OPEN ACCESS

Edited by:

Ruixi Li,

Southern University of Science and Technology, China

Reviewed by:

Caiji Gao,

South China Normal University, China Yingnan Hou,

Shanghai Jiao Tong University, China

${ }^{*}$ Correspondence:

Qiang Cai

qiang.cai@whu.edu.cn

Specialty section:

This article was submitted to

Plant Physiology,

a section of the journal

Frontiers in Plant Science

Received: 13 August 2021 Accepted: 07 September 2021 Published: 30 September 2021

Citation:

Liu G, Kang G, Wang S, Huang Y and Cai Q (2021) Extracellular Vesicles: Emerging Players in Plant Defense Against Pathogens.

Front. Plant Sci. 12:757925. doi: 10.3389/fpls.2021.757925

\section{Extracellular Vesicles: Emerging Players in Plant Defense Against Pathogens}

\author{
Guosheng Liu' ${ }^{1,2}$, Guangren Kang ${ }^{1,2}$, Shumei Wang ${ }^{3}$, Yifan Huang ${ }^{1,2}$ and Qiang Cai ${ }^{1,2 *}$ \\ 'State Key Laboratory of Hybrid Rice, College of Life Science, Wuhan University, Wuhan, China, ${ }^{2}$ Hubei Hongshan \\ Laboratory, Wuhan, China, ${ }^{3}$ Department of Microbiology and Plant Pathology, Center for Plant Cell Biology, Institute for \\ Integrative Genome Biology, University of California, Riverside, Riverside, CA, United States
}

Communication between plants and interacting microorganisms requires functional molecule trafficking, which is essential for host defense and pathogen virulence. Extracellular vesicles (EVs) are single membrane-bound spheres that carry complex cargos, including lipids, proteins, and nucleic acids. They mediate cell-to-cell communication via the transfer of molecules between cells. Plant EVs have been isolated from many plant species and play a prominent role in immune system modulation and plant defense response. Recent studies have shown that plant EVs are emerging players in cross-kingdom regulation and contribute to plant immunity by mediating the trafficking of regulatory small RNA into pathogens, leading to the silencing of pathogen virulence-related genes. This review summarizes the current understanding of plant EV isolation technologies, the role of plant EVs in plant immunity, and the mechanism of plant EV biogenesis, as well as approaches for how these findings can be developed into innovative strategies for crop protection.

Keywords: extracellular vesicles, cell-to-cell communication, plant immunity, cross-kingdom RNA interference, endomembrane trafficking

\section{INTRODUCTION}

Numerous plant pathogens, including bacteria, fungi, and nematodes, are responsible for many plant diseases, which reduce the yield and quality of agricultural production worldwide every year (Fisher et al., 2012; Savary et al., 2019). Exploring the interaction between plants and pathogens is conducive to plant disease control and agricultural production. Plants and pathogens secrete multitudes of molecules into the extracellular environment for cross-border communication, which is crucial to plant defense and pathogen virulence (Delaunois et al., 2014; Toruno et al., 2016). Based on our current understanding, extracellular vesicles (EVs) represent a major way to achieve this communication (Cai et al., 2021).

EVs are single membrane-bound heterogeneous spheres that are released by cells into the extracellular space (Colombo et al., 2014). They contain a diverse variety of enclosed bioactive cargos, such as proteins, nucleic acids, and metabolites (Colombo et al., 2014). EVs are currently categorized as exosomes, microvesicles, and apoptosis-derived vesicles on the basis of their origins and sizes (van Niel et al., 2018). Exosomes, which have diameters ranging from $30 \mathrm{~nm}$ to $150 \mathrm{~nm}$, are derived from multivesicular bodies (MVBs) after fusing with the plasma membrane to release their intraluminal vesicles (ILVs; Colombo et al., 2014). Microvesicles normally refer 
to $150-1,000 \mathrm{~nm}$ vesicles, which are shed from the plasma membrane during cell stress (Heijnen et al., 1999; Colombo et al., 2014; van Niel et al., 2018). Apoptosis-derived vesicles, which are characterized by their large size range of 30-10,000 nm, result from cell apoptosis (Atkin-Smith et al., 2015).

In the 1980s, EVs were initially thought to be a disposal mechanism for waste removal from cells (Johnstone et al., 1987). However, decades of studies have shown that numerous active molecules are transported by EVs and are featured in various biological processes, including cellular communication, immune response, antigen presentation, and cancer cell migration (Colombo et al., 2014). The latest studies have indicated that plant-released EVs play a major role in transboundary communication between plants and pathogens (Cai et al., 2018b, 2019, 2021). However, owing to the limitations of EV isolation methods, the research on plant EVs is only beginning.

\section{PLANT EVs}

EVs widely exist in eukaryotes, and numerous studies have shown that in animals, EVs are heterogeneous groups that encompass diverse subclasses and perform different functions (Kowal et al., 2016; Jeppesen et al., 2019). In plants, EVs have been isolated and purified from apoplastic washing fluid (AWF) collected from leaves and seeds or from pollen germination media (Cai et al., 2021; Table 1). At least three different subtypes of EVs have been characterized in Arabidopsis by taking advantage of their markers: tetraspanin (TET) 8, penetration 1 (PEN1), and exocyst subunit Exo70 family protein (Exo70) E2 (Wang et al., 2010; Rutter and Innes, 2017; Cai et al., 2018b; Figure 1).

Animal TETs, such as CD9, CD81, and CD63, are highly enriched in the membranes of exosomes and therefore serve as exosome markers (Escola et al., 1998; Simpson et al., 2012; Andreu and Yanez-Mo, 2014; Kowal et al., 2016). TET8 and TET9 are plant homologs of animal TET proteins (Cai et al., 2018b). TET8 and TET9-positive vesicles partially colocalize with the MVB marker Rab5-like guanosine triphosphatase ARA6 (also known as RABF1) inside of cells, and TET8positive EVs have been observed outside of cells (Cai et al., 2018b; He et al., 2021). Thus, TET8-positive EVs are considered as plant exosomes. Furthermore, evidence has shown that the expression of TET8 can be induced by Botrytis cinerea infection, and TET8-positive vesicles have been observed to accumulate at $B$. cinerea infection sites (Cai et al., 2018b), showing that TET8-associated EVs are involved in response to pathogen attack.

PEN1 has been previously shown to mediate trafficking between the Golgi complex and the plasma membrane (Kwon et al., 2008). PEN1 is also present in EVs induced by bacterial pathogen infection or salicylic acid treatment (Rutter and Innes, 2017). The secretion of PEN1 is dependent on ADP ribosylation factor-GTP exchange factor GNOM (Nielsen et al., 2012), and PEN1 does not colocate with ARA6, indicating that the biogenic pathway of TET8-positive EVs is different from that of PEN1-positive EVs (He et al., 2021). Moreover, EVs isolated from transgenic plants co-express two fluorescence-tagged fusion proteins, TET8-GFP and mCherry-PEN1, and display two distinct GFP-labeled and mCherry-labeled EVs (He et al., 2021). These characteristics confirm that PEN1-positive EVs and TET8-positive EVs are two subtypes of EVs (He et al., 2021).

Exocyst-positive organelle (EXPO) is a novel organelle that is identified by using live cell imaging and immunogold labeling in plants (Wang et al., 2010; Ding et al., 2014). Although EXPO and autophagosomes are bilayer structures, they do not co-locate with each other except in vacuoles upon autophagic induction (Wang et al., 2010; Lin et al., 2015). EXPO has been observed to fuse with the plasma membrane and deliver Exo70E2-positive EVs into the extracellular space. The secretory pathway of Exo70E2 is independent of MVB pathways, and EXPO is unaffected by secretory and endocytosis inhibitors in protoplasts (Wang et al., 2010; Ding et al., 2014).

\section{TECHNIQUES FOR PLANT EV ISOLATION}

The isolation of plant EVs remains a challenge (Liu et al., 2020b). In contrast to animal EVs, which are isolated from biofluids, plant EVs are isolated from AWF (Colombo et al., 2014). Currently, a simple well-established infiltrationcentrifugation method is widely used for plant AWF collection (Wang et al., 2005; Sanmartin et al., 2007; Hatsugai et al., 2009; O'Leary et al., 2014). The detached leaf protocol is the ideal method for collecting AWF before EV isolation (O'Leary et al., 2014; Madsen et al., 2016; Cai et al., 2018b; He et al., 2021). This protocol has the merit of the removal of the distinct proximal (petiole) parts of leaves. This approach could remove irrelevant RNAs in the phloem stream (Zhang et al., 2009; Liu and Chen, 2018). In addition, the leaves are supported and unlikely to be squeezed with each other during centrifugation (He et al., 2021).

Differential centrifugation is commonly used for EV isolation from plants (Rutter and Innes, 2017; Cai et al., 2018b; Liu et al., 2020b; He et al., 2021). In this method, dead cells, cell debris, and large vesicles are removed through low-velocity centrifugation at $2,000 \times g$ and $10,000 \times g$, and the separation rate is then progressively increased to $100,000 \times g$ to pellet small plant EVs (Prado et al., 2014; Cai et al., 2018b; Liu et al., 2020b; He et al., 2021). However, the low centrifugal force of $40,000 \times g$ has also been used for the final pelleting of EVs derived from Arabidopsis and sunflower seeds and seedlings (Regente et al., 2009, 2017; Rutter and Innes, 2017). Notably, the separation efficiency for the isolation of TET8positive EVs obtained by centrifugation at $100,000 \times g$ is much higher than that obtained by centrifugation at $40,000 \times g(\mathrm{He}$ et al., 2021).

Given that plant EVs are heterogeneous populations of nanosized membrane vesicles, researchers have developed additional separation methods based on EV density and specific EV markers. Currently, the most common and practical methods for separating the subtypes of plant EVs are density 
TABLE 1 | List of the protein markers, confirmed cargoes, isolation methods, and biological functions of various EVs isolated from plants.

\begin{tabular}{|c|c|c|c|c|c|c|c|}
\hline Plant & EV marker & EV Cargo & Function & Isolation method & $\begin{array}{l}\text { Method } \\
\text { advantages }\end{array}$ & $\begin{array}{l}\text { Method } \\
\text { disadvantages }\end{array}$ & Ref. \\
\hline \multirow[t]{3}{*}{ Arabidopsis } & TET8 & sRNAs, RBPs & $\begin{array}{l}\text { Transport sRNAs } \\
\text { to } B \text {. cinerea to } \\
\text { silence virulence } \\
\text { genes }\end{array}$ & $\begin{array}{l}\text { Differential } \\
\text { centrifugation }\end{array}$ & $\begin{array}{l}\text { Enable isolation } \\
\text { from large volumes }\end{array}$ & $\begin{array}{l}\text { Unable to separate } \\
\text { different EVs }\end{array}$ & $\begin{array}{l}\text { Cai et al., 2018b, } \\
\text { He et al., } 2021\end{array}$ \\
\hline & & & & $\begin{array}{l}\text { Density gradient } \\
\text { centrifugation }\end{array}$ & $\begin{array}{l}\text { Enable separate } \\
\text { EV sub-types }\end{array}$ & $\begin{array}{l}\text { Unable to separate } \\
\text { EVs with similar } \\
\text { density }\end{array}$ & \\
\hline & & & & $\begin{array}{l}\text { Immunoaffinity } \\
\text { capture }\end{array}$ & $\begin{array}{l}\text { High purity, high } \\
\text { selectivity }\end{array}$ & $\begin{array}{l}\text { Require specific } \\
\text { antibodies }\end{array}$ & \\
\hline \multirow[t]{2}{*}{ Arabidopsis } & PEN1 & PATL1, PATL2 & $\begin{array}{l}\text { Enriched in plant } \\
\text { defense } \\
\text { components }\end{array}$ & $\begin{array}{l}\text { Differential } \\
\text { centrifugation }\end{array}$ & $\begin{array}{l}\text { Enable isolation } \\
\text { from large volumes }\end{array}$ & $\begin{array}{l}\text { Unable to separate } \\
\text { different EVs }\end{array}$ & $\begin{array}{l}\text { Rutter and Innes, } \\
2017\end{array}$ \\
\hline & & & & $\begin{array}{l}\text { Density gradient } \\
\text { centrifugation }\end{array}$ & $\begin{array}{l}\text { Enable separate } \\
\text { EV sub-types }\end{array}$ & $\begin{array}{l}\text { Unable to separate } \\
\text { EVs with similar } \\
\text { density }\end{array}$ & \\
\hline Sunflower & nd & $\begin{array}{l}\text { PMR5, GDSL, } \\
\text { Lectins }\end{array}$ & $\begin{array}{l}\text { Antifungal activity, } \\
\text { enriched in cell } \\
\text { wall enzymes }\end{array}$ & $\begin{array}{l}\text { Differential } \\
\text { centrifugation }\end{array}$ & $\begin{array}{l}\text { Enable isolation } \\
\text { from large volumes }\end{array}$ & $\begin{array}{l}\text { Unable to separate } \\
\text { different EVs }\end{array}$ & $\begin{array}{l}\text { Regente et al., } \\
\text { 2017, de la Canal } \\
\text { and Pinedo, } 2018\end{array}$ \\
\hline \multirow[t]{2}{*}{ Olive } & $\begin{array}{l}\text { Ole e1, Ole e11, } \\
\text { Ole e12 }\end{array}$ & PCBER, GADPH & $\begin{array}{l}\text { Secreted during } \\
\text { pollen germination } \\
\text { and pollen tube } \\
\text { growth }\end{array}$ & $\begin{array}{l}\text { Differential } \\
\text { centrifugation }\end{array}$ & $\begin{array}{l}\text { Enable isolation } \\
\text { from large volumes }\end{array}$ & $\begin{array}{l}\text { Unable to separate } \\
\text { different EVs }\end{array}$ & Prado et al., 2014 \\
\hline & & & & $\begin{array}{l}\text { Density gradient } \\
\text { centrifugation }\end{array}$ & $\begin{array}{l}\text { Enable separate } \\
\text { EV sub-types }\end{array}$ & $\begin{array}{l}\text { Unable to separate } \\
\text { EVs with similar } \\
\text { density }\end{array}$ & \\
\hline N. benthamiana & nd & HSP70, AGO2 & $\begin{array}{l}\text { Release virus } \\
\text { components }\end{array}$ & $\begin{array}{l}\text { Differential } \\
\text { centrifugation }\end{array}$ & $\begin{array}{l}\text { Enable isolation } \\
\text { from large volumes }\end{array}$ & $\begin{array}{l}\text { Unable to separate } \\
\text { different EVs }\end{array}$ & $\begin{array}{l}\text { Movahed et al., } \\
2019\end{array}$ \\
\hline
\end{tabular}

AGO2, Argonaute 2; GADPH, glyceraldehyde 3-phosphate dehydrogenase; HSP70, heat shock 70kDa protein; n.d., no date (reference materials); PATL1, PATELLIN 1; PATL2, PATELLIN 2; PEN1, Penetration 1; RBPS, RNA binding protein; PCBER, phenylcoumaran benzylic ether reductase PT1; PMR5, powdery mildew resistance protein 5; sRNA, small RNA; TET8, tetraspanin 8.

gradient centrifugation and immunoaffinity capture-based techniques (Rutter and Innes, 2017; He et al., 2021). In density gradient centrifugation, sucrose and iodixanol are used as the classical media (Kowal et al., 2016; Paolini et al., 2016; Rutter and Innes, 2017; He et al., 2021). Through separation by density gradient centrifugation, TET8-positive exosomes are enriched in the gradient fraction of approximately 1.12$1.19 \mathrm{~g} \mathrm{ml}^{-1}$, whereas PEN1-positive EVs are enriched in the gradient fraction of 1.029-1.056 $\mathrm{g} \mathrm{ml}^{-1}$ (Rutter and Innes, 2017; He et al., 2021).

Although density gradient centrifugation can yield highquality EVs, different EV types may have similar physical properties. Separating EVs by immunoaffinity with EV markers may be needed. Immunoaffinity can capture specific EV subtypes with high quality at a low cost and within a short period. The activity of captured EVs is preserved to a great extent, and the captured EVs can be directly used for downstream analysis after elution (Tauro et al., 2012; Li et al., 2019). Recently, He et al. developed an immunoaffinity capture method for plant EVs (He et al., 2021). In this method, agarose beads conjugated with TET8 antibodies can efficiently isolate TET8positive EVs (He et al., 2021). By using this method, several RNA-binding proteins (RBPs), such as argonaute 1 (AGO1), annexin1, and RNA helicases (RH11 and RH37), have been identified in TET8-positive EVs (He et al., 2021).

\section{EVs IN CROSS-KINGDOM RNA INTERFERENCE}

sRNAs are short noncoding molecules that induce RNA interference (RNAi; Baulcombe, 2004). RNAi is a regulatory mechanism for gene expression that is conserved throughout the domain Eukarya. During microbial infection, host sRNA functions endogenously by regulating gene expression to balance plant immunity and growth (Katiyar-Agarwal and Jin, 2010). Emerging studies have shown that bidirectional sRNA trafficking between hosts and interacting microorganisms/pests silence target genes in trans in a mechanism referred to as crosskingdom RNAi (Cai et al., 2018a; Huang et al., 2019). For example, Arabidopsis delivers small interfering RNAs (siRNAs), including phased secondary siRNAs, into interacting $B$. cinerea cells, inducing the silencing of fungal genes that are involved in vesicle trafficking pathways (Cai et al., 2018b). Cross-kingdom RNA trafficking from the host into the pathogen to induce the silencing of corresponding pathogenic genes has also been observed in other plant pathosystems, such as in the cottonVerticillium dahliae, and wheat-Fusarium graminearum systems (Zhang et al., 2016; Jiao and Peng, 2018). Pathogens also transfer sRNAs to plant hosts as effectors to promote infection and plant defense (Cai et al., 2021). The trafficking of $B$. cinerea sRNA into Arabidopsis cells suppresses host immune 


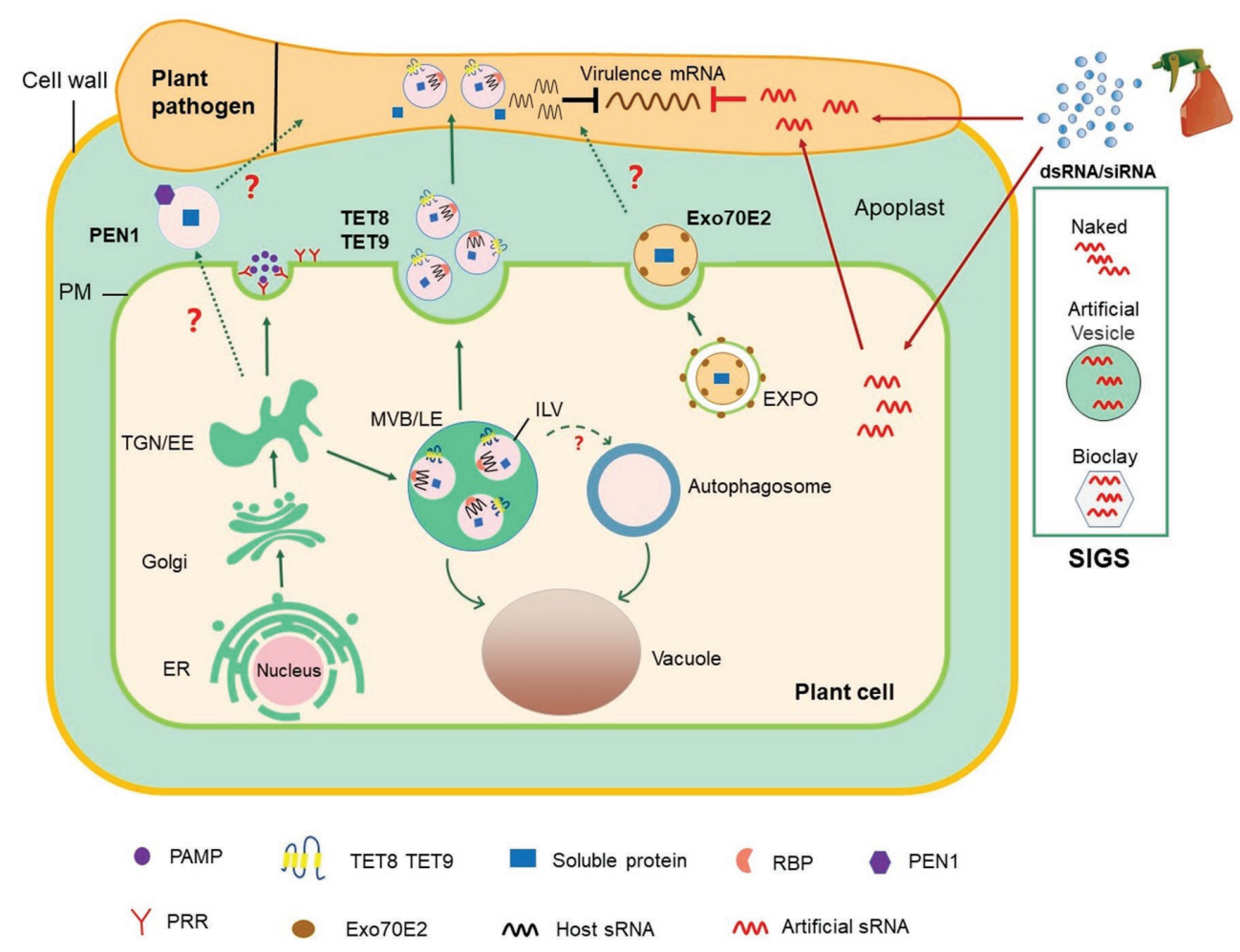

FIGURE 1 | Roles of EV-mediated RNAi in plant-microbial interactions and plant protection. The conventional secretion pathway delivers PAMPs to the extracellular space or transport surface PRRs to PM. In unconventional secretion pathway, MVBs release TET8/9-positive EVs, which contain defense proteins and host-derived SRNAs, into the extracellular space. TET8/9-positive EVs contain a variety of RBPs, including AGO1, RHs, and ANNs, which load sRNAs into EVs. PEN1-positive EVs are secreted into the extracellular space with an unknown mechanism (marked with ? in the Figure). EXPO is a novel bilayer membrane organelle that fuses with PM to produce another subtype of EVs. Whether PEN1-positive and EXPO-positive EVs contribute to cross-kingdom RNAi between plant and pathogens (marked with ? in the figure) is unknown. On the basis of the knowledge on cross-kingdom RNAi, the spray application of dsRNAs and sRNAs that target pathogen genes can potentially control plant diseases. In SIGS approaches, dsRNAs are applied exogenously or carried by nanocarriers, such as BioClay and artificial vesicles. Exogenous RNAs can either be directly internalized into fungal cells or indirectly via passage through plant cells before transport into fungal cells. dsRNA, doublestranded RNA; EE, early endosome; ER, endoplasmic reticulum; EVs, extracellular vesicles; EXPO, exocyst-positive organelle; MVB/LE, multivesicular body/late endosome; ILV, intraluminal vesicle; PAMP, pathogen-associated molecular pattern; PEN1, Penetration 1; PM, plasma membrane; PRR, pattern recognition receptor; RBPs, RNA binding protein; SIGS, spray-induced gene silencing; siRNA, small interfering RNA; sRNA, small RNA; TGN, trans-Golgi network.

genes by using the host RNAi machinery component AGO1 (Weiberg et al., 2013). Moreover, the translocated sRNAs of Hyaloperonospora arabidopsidis associate with the host Arabidopsis AGO1/RISC to regulate plant host defense genes (Dunker et al., 2020). Puccinia striiformis f. sp. Tritici (Pst), one of the most destructive pathogens of wheat (Triticum aestivum L.), produces the microRNA-like RNA 1 to silence the wheat pathogenesisrelated 2 gene, which impairs wheat defenses during wheat-Pst interactions (Wang et al., 2017).

Further studies have revealed that cross-boundary sRNA trafficking depends on EVs (Buck et al., 2014; Cai et al., 2018b, 2021; Hou et al., 2019). sRNA trafficking between cells via EVs has been studied in mammalian cells (Valadi et al., 2007; Colombo et al., 2014). In animal systems, the parasite nematode Heligmosomoides polygyrus delivers sRNAs via EVs into mouse gut epithelial cells to modulate host innate immunity (Buck et al., 2014). However, whether hosts use EVs to send sRNAs into interacting pathogen/parasite cells has long remained unclear. A recent study has revealed that more than $70 \%$ of the Arabidopsis sRNAs transported into B. cinerea cells are present in plant EVs, indicating that in plants, EV-mediated transport is one of the major pathways for the cross-kingdom trafficking of sRNA (Cai et al., 2018b). Furthermore, Arabidopsis tet8/tet9 double mutants transfer less sRNAs into fungal cells and enhance susceptibility to $B$. cinerea challenge, suggesting that plant EVs contribute to plant immunity by cross-kingdom RNAi (Cai et al., 2018b). This finding was supported by the recent study where in Arabidopsis-derived secondary siRNAs were found in EVs and likely silenced target genes in $P$. capsici during natural infection (Hou et al., 2019). However, how sRNAs are selectively loaded into EVs during vesicle biogenesis is poorly understood. A recent study illustrated that plant TET8 positive-EVs contain a variety of RBPs, including AGO1, RHs, and ANNs (He et al., 2021). These RBPs bind to sRNAs to load sRNAs into plant EVs (He et al., 2021). Interestingly, AGO1, RH11, and RH37 selectively load sRNAs into EVs, whereas ANN1/2 bind to RNA nonspecifically, indicating that they contribute to stabilizing sRNAs in EVs (He et al., 2021).

Fungal and bacterial RNA cargoes in EVs have been shown to play a pivotal role in animal host cells by regulating gene 
expression and immunity (Munhoz da Rocha et al., 2020). For example, the fatal human fungal pathogen Cryptococcus gattii secretes EVs for transferring RNAs to host cells as virulence factors (Bielska et al., 2018). In addition, sRNAs cargos have been detected in EVs derived from several bacterial pathogens, such as Aggregatibacter actinomycetemcomitans, Porphyromonas gingivalis, Pseudomonas aeruginosa, and Treponema denticola (Koeppen et al., 2016; Choi et al., 2017). These Gram-negative bacteria-derived EVs are released from the outer membranes and are also named as outer membrane vesicles (OMVs; Nahui Palomino et al., 2021). The bidirectional translocation of sRNAs has been observed in plant-pathogen interactions (Wang et al., 2016). However, studies on RNA in EVs derived from plant fungal and bacterial pathogens have not been reported. The fungal pathogen $B$. cinerea and the oomycete pathogen $H$. arabidopsidis have recently been shown to deliver sRNAs into plant host cells (Weiberg et al., 2013; Dunker et al., 2020). Further studies are needed to determine whether the eukaryotic plant pathogens that deliver RNA species into hosts require EVs.

\section{BIOGENESIS AND SECRETION OF PLANT EVs}

Exosomes are derived from MVB trafficking and finally fuse with the plasma membrane (Pegtel and Gould, 2019). The perimeter membrane of the late endosome buds inward to the endosome lumen, forming ILVs, which lead to the formation of multivesicular endosomes (van Niel et al., 2018; Mathieu et al., 2019). Therefore, they are also considered as MVBs. Two major mechanisms of ILV formation exist in animals: Endosomal Sorting Complex Required for Transport (ESCRT)-mediated pathway and ceramidemediated pathway (van Niel et al., 2018; Mathieu et al., 2019). Mechanisms that drive the mobilization of secretory MVBs and fusion with the plasma membrane require the participation of Rab family proteins (Rab11, Rab35, and Rab27), and SNARE family proteins (vesicle-associated membrane protein 7 and YKT6 V-SNARE homolog; Hsu et al., 2010; Ostrowski et al., 2010; Kowal et al., 2014; Tian et al., 2020; Ferro et al., 2021). In fact, in animal cells, MVBs also fuse with autophagosomes and further form amphisomes following the release of EVs containing autophagy components (Klionsky et al., 2014; Jeppesen et al., 2019). However, the mechanism of the formation of plant EVs remains unclear. The fusion of MVBs with the plasma membrane during plant biotic stress responses or plant growth has been demonstrated (An et al., 2006a,b; Wang et al., 2011; Cai et al., 2018b). During the infection of barley (Hordeum vulgare) by powdery mildew fungus (B. graminis f. sp. hordei), multivesicular compartments fuse with the plasma membrane and then release paramural vesicles that are similar to exosomes and may participate in papilla deposition (An et al., 2006a,b). In lily and tobacco pollen tubes, vacuolar sorting receptors (VSRs) mediate the vacuolar transport of soluble cargoes via MVBs and localize to the plasma membrane, indicating that VSR proteins have an additional role in mediating protein transport to the plasma membrane (Wang et al., 2011). A recent study has demonstrated that sphingolipids in plant EVs mainly comprise pure glycol inositol phosphate ceramides (GIPCs; Liu et al., 2020a). Furthermore, the Arabidopsis tet8 mutant has a low amount of cellular GIPCs and secretes few EVs, suggesting that GIPCs may play a part in the biogenesis of EVs (Liu et al., 2020a).

\section{NOVEL EV- AND RNAi-BASED TOOLS FOR CROP PROTECTION}

Host-induced gene silencing (HIGS) is an effective strategy for developing resistant varieties by expressing double-stranded RNAs (dsRNAs) targeting pathogen genes in plants to induce the silencing of essential pathogen genes (Nowara et al., 2010). For example, expressing a dsRNA targeting the Fusarium verticillioides gene gus enhances tobacco resistance to fungal pathogens (Tinoco et al., 2010). Silencing the Magnaporthe oryzae transcription factor MoAP1 by HIGS in transgenic rice leads to improved blast disease resistance (Guo et al., 2019). HIGS has also been used to inhibit the growth of western corn rootworm (D. virgifera LeConte) in corn and rust fungi (Puccinia triticina) in wheat (Yin et al., 2011; Bolognesi et al., 2012; Panwar et al., 2013). This strategy also can effectively prevent and control root diseases that are difficult to control via traditional chemical control. An example of such a disease is cotton Verticillium wilt caused by V. dahlia (Wang et al., 2016). Recently, SmartStax Pro, a genetically modified organism (GMO) crop developed by Bayer on the basis of RNAi technologies against insect pests, has been approved by the US government (Rosa et al., 2018). This GMO can express dsRNA corresponding to rootworm Snf7 messenger RNA (mRNA; Rosa et al., 2018).

However, HIGS has some disadvantages: (1) Transgenic expression is not always stable and is inhibited or silenced after generations; (2) uncertainty about government approvals and public concerns about GMOs; (3) currently, many plants cannot be genetically modified by transgenic technology. Recent studies have indicated that spraying dsRNAs or sRNAs that target crucial pathogen genes can provide efficient and sustainable protection to plants to solve the above problems (Figure 1). This new and innovative technology is called spray-induced gene silencing (SIGS), which has been applied to control numerous economically important plant pathogens (Wang et al., 2016; Cai et al., 2018a). For example, spraying the long noncoding dsRNA CYP3, which targets three fungal cytochrome P450 lanosterol C-14 $\alpha$-demethylases, inhibits the growth of $F$. graminearum on barley (Koch et al., 2016). Spraying dsRNA and sRNAs that target B. cinerea Dicer-like 1 and 2 on the surfaces of fruits, vegetables, and flowers can effectively inhibit gray mold disease caused by B. cinerea (Wang et al., 2016). $H$. arabidopsidis is an obligate biotrophic oocyte pathogen that induces downy mildew in Arabidopsis. The application of exogenous sRNA or dsRNAs synthesized in vitro and targeting the conserved cellulose synthase A3 gene of $H$. arabidopsidis impairs spore germination and hence prevents the infection of Arabidopsis (Bilir et al., 2019).

The success of SIGS for plant disease management is largely determined by RNA uptake efficiency, which varies among different 
pathogens. Some eukaryotic microbes, including B. cinerea, Rhizoctonia solani, Aspergillus niger, and V. dahliae, could uptake environmental RNA efficiently, whereas RNA uptake is modest in Trichoderma virens, undetectable in Colletotrichum gloeosporioides, and limited in P. infestans (Qiao et al., 2021). The efficiency of SIGS application also depends on RNA stability in the environment. Many studies have shown that EVs can protect sRNAs from degradation in the environment and have high uptake efficiency by host cells (Colombo et al., 2014; Liu et al., 2020b). Liposomes, also called artificial vesicles, are spherical vesicles that are encased by a lipid bilayer with nontoxicity, low immunogenicity, and high biocompatibility (Tseng et al., 2009). Liposomes and lipid-based nanoparticles are the most advanced and potent delivery systems for RNA drugs, which can effectively deliver siRNAs to their targets, reducing total siRNA doses and thus attenuating their potential toxicity (Ickenstein and Garidel, 2019). For therapeutic applications in mammalian systems, EVs and artificial vesicles not only carry RNAs but also carry other beneficial molecules, such as celastrol and curcumin for anticancer therapy and gold nanoparticles for improved imaging (Aqil et al., 2016; Meng et al., 2020). Current studies in the field of plant-pathogen interaction have shown that plants use EVs to transport sRNAs into their fungal pathogens (Cai et al., 2018b). Given this new knowledge, incorporating RNAs into artificial vesicles/liposomes or nanoparticles will likely facilitate RNA delivery via SIGS approaches to protect RNAs from degradation or water rinsing (Figure 1). Indeed, dsRNA loaded on nanoparticles, such as nontoxic layered double hydroxide clay nanosheets (BioClay), can continuously protect plants against virus even after 30 days of spraying (Mitter et al., 2017). Thus, the use of EVs and nanoparticles as carriers of RNAi for crop protection is a promising field in the future.

\section{CONCLUDING REMARKS AND FUTURE PERSPECTIVES}

In the past few decades, EVs have been considered as effective carriers for intercellular communication in prokaryotes and eukaryotes due to their capability to transfer proteins, lipids, nucleic acids, and other biologically active substances, thus affecting a variety of the physiological and pathological functions of their receptor cells (Colombo et al., 2014). In recent years, many studies have revealed that EVs are crucial tools for communication between plants and pathogens and execute considerable functions in host immunity and pathogen virulence (Cai et al., 2021). Plant cells secrete EVs containing sRNAs into fungal cells to induce the silencing of fungal genes that are critical for pathogenicity (Cai et al., 2018b). Recent studies have shown that EVs from the cotton pathogen Fusarium oxysporum f. sp. vasinfectum induce a phytotoxic response in plants (Bleackley et al., 2019). In addition, OMVs derived from bacteria play a vital part in biofilm formation, virulence, and plant immune regulation during plant-bacterium interactions (Katsir and Bahar, 2017). However, reports on RNAs in EVs derived from plant pathogens or interacting microbes still not exist. Further studies need to be performed to determine whether plant pathogen EVs contain RNAs that are functional within host plant cells.
RNA interference is a conserved biological defense mechanism and is thus an effective method for controlling a variety of pests and pathogens (Majumdar et al., 2017; Zhang et al., 2017). sRNAs can move between interacting organisms, inducing gene silencing in each other, in a process called cross-kingdom RNAi (Cai et al., 2018a). HIGS by the transgenic expression of pathogen dsRNA is thus expected to be an important disease control method (Rosa et al., 2018). Given the disadvantages of transgenic approaches, sRNAs can be directly sprayed on host plants or postharvest products to silence target pathogenic genes in an approach known as SIGS (Cai et al., 2018a). Such an approach can provide effective and sustainable protection to plants. However, the success of SIGS for plant disease management largely depends on the efficiency of dsRNA uptake, which varies among different pathogens (Qiao et al., 2021). The stability of RNA in the environment also affects the efficiency of SIGS (Mitter et al., 2017). Strikingly, EVs can protect sRNAs from environmental degradation and can be efficiently absorbed by host cells (Colombo et al., 2014; Liu et al., 2020b). Artificial vesicles and lipid-based nanoparticles can effectively send siRNAs to their targets at low overall doses and with low potential toxicity (Ickenstein and Garidel, 2019). Therefore, the combined use of artificial vesicles/nanoparticles and SIGS is a desirable method for crop protection.

The current research on the role of EVs in plant immune response remains in its infancy. Many momentous molecular mechanisms of EVs, such as biogenesis (MVB formation and secretion), and receptor cell absorption, remain to be further investigated. In addition to siRNAs, whether long noncoding RNAs and mRNAs exist in plant EVs is unclear. Additional plant EV cargos and their functions require urgent confirmation. In addition, due to the heterogeneity of EVs and their complex functions, further isolating different EVs and determining the effect of each type of EV are very meaningful. He et al. developed an immunoaffinity capture-based technique that can accurately isolate the subtypes of specific EVs (He et al., 2021). Different types of EVs can be obtained via direct immunoaffinity capture by using specific antibodies to determine the components and subsequent functions of each kind of vesicle. Studying and identifying additional EV markers in plants is the most current urgent task. Undoubtedly, EVs are a treasured land to seek.

\section{AUTHOR CONTRIBUTIONS}

GL reviewed the published data and prepared the initial draft. GK, SW, and YH collaborated in manuscript preparation. QC designed the structure of the review, supervised the manuscript preparation and revised and finalized the manuscript. All authors read and approved the final manuscript.

\section{FUNDING}

Work in the Q.C. laboratory was supported by grants from the National Natural Science Foundation of China (32070288). 


\section{REFERENCES}

An, Q., Ehlers, K., Kogel, K. H., Van Bel, A. J., and Huckelhoven, R. (2006a). Multivesicular compartments proliferate in susceptible and resistant MLA12-barley leaves in response to infection by the biotrophic powdery mildew fungus. New Phytol. 172, 563-576. doi: 10.1111/j.1469-8137.2006. 01844.x

An, Q., Huckelhoven, R., Kogel, K. H., and Van Bel, A. J. (2006b). Multivesicular bodies participate in a cell wall-associated defence response in barley leaves attacked by the pathogenic powdery mildew fungus. Cell. Microbiol. 8, 1009-1019. doi: 10.1111/j.1462-5822.2006.00683.x

Andreu, Z., and Yanez-Mo, M. (2014). Tetraspanins in extracellular vesicle formation and function. Front. Immunol. 5:442. doi: 10.3389/fimmu.2014.00442

Aqil, F., Kausar, H., Agrawal, A. K., Jeyabalan, J., Kyakulaga, A. H., Munagala, R., et al. (2016). Exosomal formulation enhances therapeutic response of celastrol against lung cancer. Exp. Mol. Pathol. 101, 12-21. doi: 10.1016/j. yexmp.2016.05.013

Atkin-Smith, G. K., Tixeira, R., Paone, S., Mathivanan, S., Collins, C., Liem, M., et al. (2015). A novel mechanism of generating extracellular vesicles during apoptosis via a beads-on-a-string membrane structure. Nat. Commun. 6:7439. doi: $10.1038 /$ ncomms 8439

Baulcombe, D. (2004). RNA silencing in plants. Nature 431, 356-363. doi: 10.1038 /nature02874

Bielska, E., Sisquella, M. A., Aldeieg, M., Birch, C., O'donoghue, E. J., and May, R. C. (2018). Pathogen-derived extracellular vesicles mediate virulence in the fatal human pathogen Cryptococcus gattii. Nat. Commun. 9:1556. doi: 10.1038/s41467-018-03991-6

Bilir, O., Telli, O., Norman, C., Budak, H., Hong, Y., and Tor, M. (2019). Small RNA inhibits infection by downy mildew pathogen Hyaloperonospora arabidopsidis. Mol. Plant Pathol. 20, 1523-1534. doi: 10.1111/mpp.12863

Bleackley, M. R., Samuel, M., Garcia-Ceron, D., Mckenna, J. A., Lowe, R. G. T., Pathan, M., et al. (2019). Extracellular vesicles from the cotton pathogen Fusarium oxysporum f. sp. vasinfectum induce a phytotoxic response in plants. Front. Plant Sci. 10:1610. doi: 10.3389/fpls.2019.01610

Bolognesi, R., Ramaseshadri, P., Anderson, J., Bachman, P., Clinton, W., Flannagan, R., et al. (2012). Characterizing the mechanism of action of double-stranded RNA activity against western corn rootworm (Diabrotica virgifera virgifera LeConte). PLoS One 7:e47534. doi: 10.1371/journal. pone.0047534

Buck, A. H., Coakley, G., Simbari, F., Mcsorley, H. J., Quintana, J. F., Le Bihan, T., et al. (2014). Exosomes secreted by nematode parasites transfer small RNAs to mammalian cells and modulate innate immunity. Nat. Commun. 5:5488. doi: $10.1038 /$ ncomms 6488

Cai, Q., He, B. Y., and Jin, H. L. (2019). A safe ride in extracellular vesicles small RNA trafficking between plant hosts and pathogens. Curr. Opin. Plant Biol. 52, 140-148. doi: 10.1016/j.pbi.2019.09.001

Cai, Q., He, B., Kogel, K. H., and Jin, H. (2018a). Cross-kingdom RNA trafficking and environmental RNAi-nature's blueprint for modern crop protection strategies. Curr. Opin. Microbiol. 46, 58-64. doi: 10.1016/j. mib.2018.02.003

Cai, Q., He, B., Wang, S., Fletcher, S., Niu, D., Mitter, N., et al. (2021). Message in a bubble: shuttling small RNAs and proteins between cells and interacting organisms using extracellular vesicles. Annu. Rev. Plant Biol. 72, 497-524. doi: 10.1146/annurev-arplant-081720-010616

Cai, Q., Qiao, L. L., Wang, M., He, B. Y., Lin, F. M., Palmquist, J., et al. (2018b). Plants send small RNAs in extracellular vesicles to fungal pathogen to silence virulence genes. Science 360, 1126-1129. doi: 10.1126/science. aar4142

Choi, J. W., Kim, S. C., Hong, S. H., and Lee, H. J. (2017). Secretable small RNAs via outer membrane vesicles in periodontal pathogens. J. Dent. Res. 96, 458-466. doi: 10.1177/0022034516685071

Colombo, M., Raposo, G., and Thery, C. (2014). Biogenesis, secretion, and intercellular interactions of exosomes and other extracellular vesicles. Annu. Rev. Cell Dev. Biol. 30, 255-289. doi: 10.1146/annurev-cellbio101512-122326

de la Canal, L., and Pinedo, M. (2018). Extracellular vesicles: a missing component in plant cell wall remodeling. J. Exp. Bot. 69, 4655-4658. doi: 10.1093/jxb/ ery255
Delaunois, B., Jeandet, P., Clement, C., Baillieul, F., Dorey, S., and Cordelier, S. (2014). Uncovering plant-pathogen crosstalk through apoplastic proteomic studies. Front. Plant Sci. 5:249. doi: 10.3389/fpls.2014.00249

Ding, Y., Wang, J., Lai, J. H. C., Chan, V. H. L., Wang, X. F., Cai, Y., et al. (2014). Exo70E2 is essential for exocyst subunit recruitment and EXPO formation in both plants and animals. Mol. Biol. Cell 25, 412-426. doi: 10.1091/mbc.e13-10-0586

Dunker, F., Trutzenberg, A., Rothenpieler, J. S., Kuhn, S., Prols, R., Schreiber, T., et al. (2020). Oomycete small RNAs bind to the plant RNA-induced silencing complex for virulence. eLife 9:e56096. doi: 10.7554/eLife.56096

Escola, J. M., Kleijmeer, M. J., Stoorvogel, W., Griffith, J. M., Yoshie, O., and Geuze, H. J. (1998). Selective enrichment of tetraspan proteins on the internal vesicles of multivesicular endosomes and on exosomes secreted by human B-lymphocytes. J. Biol. Chem. 273, 20121-20127. doi: 10.1074/ jbc.273.32.20121

Ferro, E., Bosia, C., and Campa, C. C. (2021). RAB11-mediated trafficking and human cancers: an updated review. Biology 10:26. doi: 10.3390/ biology 10010026

Fisher, M. C., Henk, D. A., Briggs, C. J., Brownstein, J. S., Madoff, L. C., Mccraw, S. L., et al. (2012). Emerging fungal threats to animal, plant and ecosystem health. Nature 484, 186-194. doi: 10.1038/nature10947

Guo, X. Y., Li, Y., Fan, J., Xiong, H., Xu, F. X., Shi, J., et al. (2019). Host-induced gene silencing of MoAP1 confers broad-spectrum resistance to Magnaporthe oryzae. Front. Plant Sci. 10:433. doi: 10.3389/fpls.2019.00433

Hatsugai, N., Iwasaki, S., Tamura, K., Kondo, M., Fuji, K., Ogasawara, K., et al. (2009). A novel membrane fusion-mediated plant immunity against bacterial pathogens. Genes Dev. 23, 2496-2506. doi: 10.1101/gad.1825209

He, B., Cai, Q., Qiao, L., Huang, C. Y., Wang, S., Miao, W., et al. (2021). RNA-binding proteins contribute to small RNA loading in plant extracellular vesicles. Nat. Plants 7, 342-352. doi: 10.1038/s41477-021-00863-8

Heijnen, H. F. G., Schiel, A. E., Fijnheer, R., Geuze, H. J., and Sixma, J. J. (1999). Activated platelets release two types of membrane vesicles: microvesicles by surface shedding and exosomes derived from exocytosis of multivesicular bodies and alpha-granules. Blood 94, 3791-3799. doi: 10.1182/blood. V94.11.3791

Hou, Y. N., Zhai, Y., Feng, L., Karimi, H. Z., Rutter, B. D., Zeng, L. P., et al. (2019). A phytophthora effector suppresses trans-kingdom RNAi to promote disease susceptibility. Cell Host Microbe 25, 153.e5-165.e5. doi: 10.1016/j. chom.2018.11.007

Hsu, C., Morohashi, Y., Yoshimura, S., Manrique-Hoyos, N., Jung, S. Y., Lauterbach, M. A., et al. (2010). Regulation of exosome secretion by Rab35 and its GTPase-activating proteins TBC1D10A-C. J. Cell Biol. 189, 223-232. doi: $10.1083 /$ jcb.200911018

Huang, C. Y., Wang, H., Hu, P., Hamby, R., and Jin, H. (2019). Small RNAs big players in plant-microbe interactions. Cell Host Microbe 26, 173-182. doi: 10.1016/j.chom.2019.07.021

Ickenstein, L. M., and Garidel, P. (2019). Lipid-based nanoparticle formulations for small molecules and RNA drugs. Expert Opin. Drug Deliv. 16, 1205-1226. doi: 10.1080/17425247.2019.1669558

Jeppesen, D. K., Fenix, A. M., Franklin, J. L., Higginbotham, J. N., Zhang, Q., Zimmerman, L. J., et al. (2019). Reassessment of exosome composition. Cell 177, 428.e18-445.e18. doi: 10.1016/j.cell.2019.02.029

Jiao, J., and Peng, D. (2018). Wheat microRNA1023 suppresses invasion of Fusarium graminearum via targeting and silencing FGSG_03101. J. Plant Interact. 13, 514-521. doi: 10.1080/17429145.2018.1528512

Johnstone, R. M., Adam, M., Hammond, J. R., Orr, L., and Turbide, C. (1987). Vesicle formation during reticulocyte maturation - Association of plasmamembrane activities with released vesicles (exosomes). J. Biol. Chem. 262, 9412-9420. doi: 10.1016/S0021-9258(18)48095-7

Katiyar-Agarwal, S., and Jin, H. (2010). Role of small RNAs in host-microbe interactions. Annu. Rev. Phytopathol. 48, 225-246. doi: 10.1146/annurevphyto-073009-114457

Katsir, L., and Bahar, O. (2017). Bacterial outer membrane vesicles at the plant-pathogen interface. PLoS Pathog. 13:e1006306. doi: 10.1371/journal. ppat.1006306

Klionsky, D. J., Eskelinen, E. L., and Deretic, V. (2014). Autophagosomes, phagosomes, autolysosomes, phagolysosomes, autophagolysosomes. Wait, I'm confused. Autophagy 10, 549-551. doi: 10.4161/auto.28448 
Koch, A., Biedenkopf, D., Furch, A., Weber, L., Rossbach, O., Abdellatef, E., et al. (2016). An RNAi-based control of Fusarium graminearum infections through spraying of long dsRNAs involves a plant passage and is controlled by the fungal silencing machinery. PLoS Pathog. 12:e1005901. doi: 10.1371/ journal.ppat.1005901

Koeppen, K., Hampton, T. H., Jarek, M., Scharfe, M., Gerber, S. A., Mielcarz, D. W., et al. (2016). A novel mechanism of host-pathogen interaction through sRNA in bacterial outer membrane vesicles. PLoS Pathog. 12:e1005672. doi: 10.1371/journal.ppat.1005672

Kowal, J., Arras, G., Colombo, M., Jouve, M., Morath, J. P., Primdal-Bengtson, B., et al. (2016). Proteomic comparison defines novel markers to characterize heterogeneous populations of extracellular vesicle subtypes. Proc. Natl. Acad. Sci. U. S. A. 113, E968-E977. doi: 10.1073/pnas.1521230113

Kowal, J., Tkach, M., and Thery, C. (2014). Biogenesis and secretion of exosomes. Curr. Opin. Cell Biol. 29, 116-125. doi: 10.1016/j.ceb.2014.05.004

Kwon, C., Neu, C., Pajonk, S., Yun, H. S., Lipka, U., Humphry, M., et al. (2008). Co-option of a default secretory pathway for plant immune responses. Nature 451, 835-840. doi: 10.1038/nature06545

Li, J., He, X. Q., Deng, Y. Y., and Yang, C. X. (2019). An update on isolation methods for proteomic studies of extracellular vesicles in biofluids. Molecules 24:3516. doi: 10.3390/molecules24193516

Lin, Y. S., Ding, Y., Wang, J., Shen, J. B., Kung, C. H., Zhuang, X. H., et al. (2015). Exocyst-positive organelles and autophagosomes are distinct organelles in plants. Plant Physiol. 169, 1917-1932. doi: 10.1104/pp.15.00953

Liu, L., and Chen, X. (2018). Intercellular and systemic trafficking of RNAs in plants. Nat. Plants 4, 869-878. doi: 10.1038/s41477-018-0288-5

Liu, N. J., Wang, N., Bao, J. J., Zhu, H. X., Wang, L. J., and Chen, X. Y. (2020a). Lipidomic analysis reveals the importance of GIPCs in Arabidopsis leaf extracellular vesicles. Mol. Plant 13, 1523-1532. doi: 10.1016/j. molp.2020.07.016

Liu, Y., Wu, S., Koo, Y., Yang, A., Dai, Y., Khant, H., et al. (2020b). Characterization of and isolation methods for plant leaf nanovesicles and small extracellular vesicles. Nanomedicine 29:102271. doi: 10.1016/j.nano.2020.102271

Madsen, S. R., Nour-Eldin, H. H., and Halkier, B. A. (2016). Collection of apoplastic fluids from Arabidopsis thaliana leaves. Methods Mol. Biol. 1405, 35-42. doi: 10.1007/978-1-4939-3393-8_4

Majumdar, R., Rajasekaran, K., and Cary, J. W. (2017). RNA interference (RNAi) as a potential tool for control of mycotoxin contamination in crop plants: concepts and considerations. Front. Plant Sci. 8:200. doi: 10.3389/fpls.2017.00200

Mathieu, M., Martin-Jaular, L., Lavieu, G., and Thery, C. (2019). Specificities of secretion and uptake of exosomes and other extracellular vesicles for cell-to-cell communication. Nat. Cell Biol. 21, 9-17. doi: 10.1038/ s41556-018-0250-9

Meng, W., He, C., Hao, Y., Wang, L., Li, L., and Zhu, G. (2020). Prospects and challenges of extracellular vesicle-based drug delivery system: considering cell source. Drug Deliv. 27, 585-598. doi: 10.1080/10717544. 2020.1748758

Mitter, N., Worrall, E. A., Robinson, K. E., Li, P., Jain, R. G., Taochy, C., et al. (2017). Clay nanosheets for topical delivery of RNAi for sustained protection against plant viruses. Nat. Plants 3:16207. doi: 10.1038/nplants.2016.207

Movahed, N., Cabanillas, D. G., Wan, J., Vali, H., Laliberte, J. F., and Zheng, H. Q. (2019). Turnip mosaic virus components are released into the extracellular space by vesicles in infected leaves. Plant Physiol. 180, 1375-1388. doi: 10.1104/pp.19.00381

Munhoz da Rocha, I. F., Amatuzzi, R. F., Lucena, A. C. R., Faoro, H., and Alves, L. R. (2020). Cross-kingdom extracellular vesicles EV-RNA communication as a mechanism for host-pathogen interaction. Front. Cell. Infect. Microbiol. 10:593160. doi: 10.3389/fcimb.2020.593160

Nahui Palomino, R. A., Vanpouille, C., Costantini, P. E., and Margolis, L. (2021). Microbiota-host communications: Bacterial extracellular vesicles as a common language. PLoS Pathog. 17:e1009508. doi: 10.1371/journal. ppat. 1009508

Nielsen, M. E., Feechan, A., Bohlenius, H., Ueda, T., and Thordal-Christensen, H. (2012). Arabidopsis ARF-GTP exchange factor, GNOM, mediates transport required for innate immunity and focal accumulation of syntaxin PEN1. Proc. Natl. Acad. Sci. U. S. A. 109, 11443-11448. doi: 10.1073/pnas.1117596109

Nowara, D., Gay, A., Lacomme, C., Shaw, J., Ridout, C., Douchkov, D., et al. (2010). HIGS: host-induced gene silencing in the obligate biotrophic fungal pathogen Blumeria graminis. Plant Cell 22, 3130-3141. doi: 10.1105/ tpc. 110.077040

O’Leary, B. M., Rico, A., Mccraw, S., Fones, H. N., and Preston, G. M. (2014). The infiltration-centrifugation technique for extraction of apoplastic fluid from plant leaves using Phaseolus vulgaris as an example. J. Vis. Exp. 94:52113. doi: $10.3791 / 52113$

Ostrowski, M., Carmo, N. B., Krumeich, S., Fanget, I., Raposo, G., Savina, A., et al. (2010). Rab27a and Rab27b control different steps of the exosome secretion pathway. Nat. Cell Biol. 12, 19-30. doi: 10.1038/ncb2000

Panwar, V., Mccallum, B., and Bakkeren, G. (2013). Host-induced gene silencing of wheat leaf rust fungus Puccinia triticina pathogenicity genes mediated by the Barley stripe mosaic virus. Plant Mol. Biol. 81, 595-608. doi: 10.1007/ s11103-013-0022-7

Paolini, L., Zendrini, A., Di Noto, G., Busatto, S., Lottini, E., Radeghieri, A., et al. (2016). Residual matrix from different separation techniques impacts exosome biological activity. Sci. Rep. 6:23550. doi: 10.1038/srep23550

Pegtel, D. M., and Gould, S. J. (2019). Exosomes. Annu. Rev. Biochem. 88, 487-514. doi: 10.1146/annurev-biochem-013118-111902

Prado, N., Alche Jde, D., Casado-Vela, J., Mas, S., Villalba, M., Rodriguez, R., et al. (2014). Nanovesicles are secreted during pollen germination and pollen tube growth: a possible role in fertilization. Mol. Plant 7, 573-577. doi: $10.1093 / \mathrm{mp} / \mathrm{sst} 153$

Qiao, L. L., Lan, C., Capriotti, L., Ah-Fong, A., Sanchez, J. N., Hamby, R., et al. (2021). Spray-induced gene silencing for disease control is dependent on the efficiency of pathogen RNA uptake. Plant Biotechnol. J. doi: 10.1111/ pbi.13589 [Epub ahead of print]

Regente, M., Corti-Monzon, G., Maldonado, A. M., Pinedo, M., Jorrin, J., and De La Canal, L. (2009). Vesicular fractions of sunflower apoplastic fluids are associated with potential exosome marker proteins. FEBS Lett. 583, 3363-3366. doi: 10.1016/j.febslet.2009.09.041

Regente, M., Pinedo, M., San Clemente, H., Balliau, T., Jamet, E., and De La Canal, L. (2017). Plant extracellular vesicles are incorporated by a fungal pathogen and inhibit its growth. J. Exp. Bot. 68, 5485-5495. doi: 10.1093/ jxb/erx355

Rosa, C., Kuo, Y. W., Wuriyanghan, H., and Falk, B. W. (2018). RNA interference mechanisms and applications in plant pathology. Annu. Rev. Phytopathol. 56, 581-610. doi: 10.1146/annurev-phyto-080417-050044

Rutter, B. D., and Innes, R. W. (2017). Extracellular vesicles isolated from the leaf apoplast carry stress-response proteins. Plant Physiol. 173, 728-741. doi: $10.1104 / \mathrm{pp} .16 .01253$

Sanmartin, M., Ordonez, A., Sohn, E. J., Robert, S., Sanchez-Serrano, J. J., Surpin, M. A., et al. (2007). Divergent functions of VTI12 and VTI11 in trafficking to storage and lytic vacuoles in Arabidopsis. Proc. Natl. Acad. Sci. U. S. A. 104, 3645-3650. doi: 10.1073/pnas.0611147104

Savary, S., Willocquet, L., Pethybridge, S. J., Esker, P., Mcroberts, N., and Nelson, A. (2019). The global burden of pathogens and pests on major food crops. Nat. Ecol. Evol. 3, 430-439. doi: 10.1038/s41559-018-0793-y

Simpson, R. J., Kalra, H., and Mathivanan, S. (2012). ExoCarta as a resource for exosomal research. J. Extracell. Vesicles 1:18374. doi: 10.3402/jev.v1i0.18374

Tauro, B. J., Greening, D. W., Mathias, R. A., Ji, H., Mathivanan, S., Scott, A. M., et al. (2012). Comparison of ultracentrifugation, density gradient separation, and immunoaffinity capture methods for isolating human colon cancer cell line LIM1863-derived exosomes. Methods 56, 293-304. doi: 10.1016/j. ymeth.2012.01.002

Tian, X. Y., Teng, J. L., and Chen, J. G. (2020). New insights regarding SNARE proteins in autophagosome-lysosome fusion. Autophagy, 1-9. doi: 10.1080/15548627.2020.1823124 [Epub ahead of print]

Tinoco, M. L., Dias, B. B., Dallastta, R. C., Pamphile, J. A., and Aragao, F. J. (2010). In vivo trans-specific gene silencing in fungal cells by in planta expression of a double-stranded RNA. BMC Biol. 8:27. doi: 10.1186/1741-7007-8-27

Toruno, T. Y., Stergiopoulos, I., and Coaker, G. (2016). Plant-pathogen effectors: cellular probes interfering with plant defenses in spatial and temporal manners. Annu. Rev. Phytopathol. 54, 419-441. doi: 10.1146/annurevphyto-080615-100204

Tseng, Y. C., Mozumdar, S., and Huang, L. (2009). Lipid-based systemic delivery of siRNA. Adv. Drug Deliv. Rev. 61, 721-731. doi: 10.1016/j. addr.2009.03.003

Valadi, H., Ekstrom, K., Bossios, A., Sjostrand, M., Lee, J. J., and Lotvall, J. O. (2007). Exosome-mediated transfer of mRNAs and microRNAs is a novel 
mechanism of genetic exchange between cells. Nat. Cell Biol. 9, 654-659. doi: $10.1038 /$ ncb1596

van Niel, G., D'angelo, G., and Raposo, G. (2018). Shedding light on the cell biology of extracellular vesicles. Nat. Rev. Mol. Cell Biol. 19, 213-228. doi: 10.1038/nrm.2017.125

Wang, J. A., Ding, Y., Wang, J. Q., Hillmer, S., Miao, Y. S., Lo, S. W., et al. (2010). EXPO, an exocyst-positive organelle distinct from multivesicular endosomes and autophagosomes, mediates cytosol to cell wall exocytosis in Arabidopsis and tobacco cells. Plant Cell 22, 4009-4030. doi: 10.1105/ tpc.110.080697

Wang, B., Sun, Y., Song, N., Zhao, M., Liu, R., Feng, H., et al. (2017). Puccinia striiformis f. sp. tritici microRNA-like RNA 1 (Pst-milR1), an important pathogenicity factor of Pst, impairs wheat resistance to Pst by suppressing the wheat pathogenesis-related 2 gene. New Phytol. 215, 338-350. doi: 10.1111/nph.14577

Wang, D., Weaver, N. D., Kesarwani, M., and Dong, X. N. (2005). Induction of protein secretory pathway is required for systemic acquired resistance. Science 308, 1036-1040. doi: 10.1126/science.1108791

Wang, M., Weiberg, A., Lin, F. M., Thomma, B. P., Huang, H. D., and Jin, H. (2016). Bidirectional cross-kingdom RNAi and fungal uptake of external RNAs confer plant protection. Nat. Plants 2:16151. doi: 10.1038/nplants.2016.151

Wang, H., Zhuang, X. H., Hillmer, S., Robinson, D. G., and Jiang, L. W. (2011). Vacuolar sorting receptor (VSR) proteins reach the plasma membrane in germinating pollen tubes. Mol. Plant 4, 845-853. doi: 10.1093/mp/ssr011 Weiberg, A., Wang, M., Lin, F. M., Zhao, H., Zhang, Z., Kaloshian, I., et al. (2013). Fungal small RNAs suppress plant immunity by hijacking host RNA interference pathways. Science 342, 118-123. doi: 10.1126/science.1239705

Yin, C. T., Jurgenson, J. E., and Hulbert, S. H. (2011). Development of a host-induced RNAi system in the wheat stripe rust fungus Puccinia striiformis f. sp tritici. Mol. Plant-Microbe Interact. 24, 554-561. doi: 10.1094/ MPMI-10-10-0229

Zhang, J., Khan, S. A., Heckel, D. G., and Bock, R. (2017). Next-generation insect-resistant plants: RNAi-mediated crop protection. Trends Biotechnol. 35, 871-882. doi: 10.1016/j.tibtech.2017.04.009

Zhang, S., Sun, L., and Kragler, F. (2009). The phloem-delivered RNA pool contains small noncoding RNAs and interferes with translation. Plant Physiol. 150, 378-387. doi: 10.1104/pp.108.134767

Zhang, T., Zhao, Y. L., Zhao, J. H., Wang, S., Jin, Y., Chen, Z. Q., et al. (2016). Cotton plants export microRNAs to inhibit virulence gene expression in a fungal pathogen. Nat. Plants 2:16153. doi: 10.1038/nplants.2016.153

Conflict of Interest: The authors declare that the research was conducted in the absence of any commercial or financial relationships that could be construed as a potential conflict of interest.

Publisher's Note: All claims expressed in this article are solely those of the authors and do not necessarily represent those of their affiliated organizations, or those of the publisher, the editors and the reviewers. Any product that may be evaluated in this article, or claim that may be made by its manufacturer, is not guaranteed or endorsed by the publisher.

Copyright (c) 2021 Liu, Kang, Wang, Huang and Cai. This is an open-access article distributed under the terms of the Creative Commons Attribution License (CC BY). The use, distribution or reproduction in other forums is permitted, provided the original author(s) and the copyright owner(s) are credited and that the original publication in this journal is cited, in accordance with accepted academic practice. No use, distribution or reproduction is permitted which does not comply with these terms. 